\title{
Mayores en la nube
}

\author{
José Traver Ardura \\ Universitat per a Majors, Universitat Jaume I, España \\ traverj@uji.es
}

\begin{abstract}
There is a change in information and communications systems that shows an evolution from powerful locally-based computers to remote systems clusters, called clouds, available to all users. The cloud is a set of virtual on-demand resources easily accessible for any user. A cloud offers three kind of services: Software as a Services (SaaS), Platform as a Service (PaaS) and Infrastructure as a Service (IaaS) and from the use people make of them it could be consider a public, a private, a community or a hybrid cloud. All those services must provide security and data integrity. Nowadays, there is a hype about pervasive healthcare computing using the cloud where dependent people or those with long-term chronic issues are monitored while data and medical alerts are stored and managed in the cloud. Also the use of cloud services has arisen in many universities, as in the Seniors' University at UII, where services like SaaS and IaaS have been widely introduced to students, researchers and staff, mainly due to a mutual working agreement with Google.
\end{abstract}

Keywords:seniors, cloud computing, pervasive healthcare computing, virtual systems

\section{Resumen}

La evolución en los sistemas de información y comunicación ha supuesto trasladar los equipos informáticos de gran potencia desde un lugar físicamente local para los usuarios hasta estar accesibles de forma remota en lo que se denomina la nube, es decir, agrupaciones de recursos virtuales fácilmente utilizables y accesibles bajo demanda. Los servicios que se pueden encontrar en la nube se dividen en programas (SaaS), plataformas de desarrollo (PaaS) e infraestructura (IaaS) y, según su uso, pueden ser privadas, públicas, comunitarias o híbridas. Todos estos servicios deben garantizar la seguridad y la integridad de la información. Hoy en día, existe un gran auge en la investigación médica y asistencial donde personas dependientes o enfermos crónicos son monitorizados y la gestión de sus datos y alertas médicas se realiza en la nube. También en las universidades se hace un uso importante de la nube, como en la Universidad para Mayores de la UJI, donde la implantación de servicios SaaS e IaaS es notable, gracias en parte a un acuerdo de colaboración con Google.

Palabras clave: mayores, nube, computación para asistencia médica, sistemas virtuales 


\section{Introducción}

En las últimas décadas, el uso de ordenadores para el cálculo y la manipulación de datos ha visto cómo se ha intensificado su uso gracias, en gran parte, al aumento exponencial de las capacidades que estos equipos ofrecían y a un progresivo abaratamiento de su coste de adquisición. Mucho se ha avanzado en este objetivo desde los sistemas de control utilizados a bordo de la nave del Apollo 11 con los que el hombre llegó a la luna en 1969, con un procesador de $1 \mathrm{Mhz}, 12$ Kwords de memoria ROM y 1 Kword de memoria RAM, hasta los modernos sistemas de computación paralela como el Sequoia BlueGene/Q con una potencia capaz de alcanzar $1,6 \cdot 1015$ operaciones en coma flotante por segundo (FLOPS).

Esta mejora en la potencia de cálculo siempre ha sido aprovechada por organismos gubernamentales, militares y centros de investigación en la simulación de procesos naturales como la previsión del tiempo o de seísmos, el análisis de cambios climáticos, el modelado molecular o las simulaciones físicas como túneles de viento, por citar solo algunos de los múltiples usos que se hacen de los sistemas informáticos más avanzados.

En esta carrera por la capacidad de cálculo y velocidad se ha visto una evolución desde el desarrollo de ordenadores destinados a un puesto de trabajo (aunque pudieran ocupar una habitación entera), pasando por supercomputadores más complejos y, recientemente, hacia el uso de agrupaciones (clústeres) de ordenadores preparados para trabajar paralelamente de forma masiva. En las décadas de los 80 y 90 triunfaban los grandes ordenadores diseñados específicamente para un entorno concreto y capaces de utilizar varios procesadores con gran cantidad de memoria, como los sistemas de Silicon Graphics Inc (sGI), IBM o Cray. A partir de la entrada en el siglo XXI, comienzan a popularizarse de forma común las tecnologías de computación distribuida mediante múltiples ordenadores de relativo bajo coste y con sistemas de comunicación de gran ancho de banda. Sus características hacen de los clústeres de sistemas distribuidos una mejor opción frente a los supercomputadores por su capacidad de escalar, el ahorro de costes de ad- quisición y mantenimiento o la flexibilidad en el diseño del sistema.

Hoy en día, estas tecnologías se pueden ofrecer a cualquier persona con unos conocimientos técnicos mínimos o, incluso, llegan a utilizarse sin que nos demos cuenta. La idea básica es poder ofrecer una gran cantidad de recursos informáticos a los usuarios sin la necesidad de que esos recursos residan físicamente en el mismo lugar que el usuario y que, además, se adapten al tamaño del problema que el usuario necesita resolver. Esto supone un gran cambio respecto a algunos conceptos previos existentes como el de tener que instalar programas o cambiar el ordenador cuando no dispone de suficiente potencia para realizar las tareas a las que se le somete.

\section{2. ¿Qué es eso de la nube?}

Esta evolución informática que se ha expuesto supone una breve exposición del desarrollo en la capacidad de proceso de datos de los sistemas informáticos. Si hoy en día los sistemas más potentes del mundo están formados por grandes clústeres de ordenadores y el uso de la computación distribuida a lo largo del planeta (GRID) es algo habitual, es necesario ver en qué momento aparece el concepto de nube y si se trata de algo novedoso que no existía hasta ahora o realmente son conceptos diferentes que comparten la visión de un mismo problema: reutilizar recursos y minimizar costes a los usuarios.

Para poder tratar el tema con propiedad, es necesario realizar una definición formal de lo que es la nube en el entorno de las tecnologías de la información y la comunicación (TIC). Sin embargo, haciendo un seguimiento de cómo ha ido apareciendo este concepto de nube, sus variados usos y orientaciones, se hace difícil conseguir una unificación en cuanto a su definición oficial.

Vaquero y otros (2008) hacen una búsqueda y análisis exhaustivos de las diferentes definiciones que se encuentran en la comunidad científica y empresarial respecto al uso del término nube y recogen diferentes visiones del mismo concepto. El conjunto de definiciones coinciden en que «las nubes son grandes conjuntos de recursos virtuales, como 
hardware, plataformas de desarrollo y servicios, que son fácilmente utilizables y accesibles».

De forma parecida, Mell (2009) ofrece una definición con intención de clarificar los conceptos básicos a través del National Institute of Standards and Technology (NIST) estadounidense. La definición es parecida y dice que:

La computación en la nube es un modelo para posibilitar el acceso ubicuo, conveniente, bajo demanda y de forma remota a un conjunto de recursos computacionales, como redes, almacenamiento, aplicaciones y servicios que pueden ser rápidamente aprovechados y liberados con un esfuerzo de mantenimiento mínimo o mediante la intervención de un proveedor de servicios Tic.

Las características, según se indica en el estándar del NIST, que ha de cumplir un sistema computacional para ser considerado una nube son las siguientes:

- Acceso y obtención de recursos bajo demanda. Los usuarios pueden, de forma unilateral y bajo demanda, abastecerse de recursos computacionales tales como tiempo de proceso para realizar cálculos o almacenamiento en red. Todo ello se hace sin que se requiera intervención humana con los proveedores de servicios Tic.

- Acceso por red. Las capacidades de la nube están disponibles a través de redes informáticas empleando mecanismos estándar que faciliten el uso en equipos heterogéneos, tanto equipos sencillos como otros más avanzados y potentes (teléfonos móviles, tabletas, portátiles o bien ordenadores de sobremesa).

- Conjunto de recursos disponibles. Los recursos del proveedor, tales como capacidad de proceso, memoria, almacenamiento o ancho de banda están disponibles para servir a muchos usuarios con diferentes asignaciones físicas o virtuales de los mismos de acuerdo a la demanda concreta de cada uno. El usuario, por lo general, no tiene un control exacto de dónde se encuentran los recursos que utiliza en la nube pero tiene algunas nociones básicas, como el país o el centro de datos donde se aloja un determinado recurso.

- Rapidez en adaptación a los cambios. Las capacidades de la nube pueden ser fácilmente utili- zadas y liberadas, de forma automática en algunos casos, para escalar de forma ágil y atender una demanda cambiante.

- Servicio mensurable. Los sistemas en la nube controlan y optimizan los recursos mediante sus propios sistemas de medición de capacidades, lo que permite el control del consumo de los recursos, conocer el empleo que se hace de ellos, monitorizar los casos de usos, etc

Por otra parte, además de estas cinco características, Dillon (2010) amplía la definición del estándar del NisT para especificar tres categorías de servicios que puede aportar la nube. Se pueden encontrar servicios de programas, plataformas e infraestructuras, e incluso hay quien separa un cuarto servicio derivado de la infraestructura que representa el tratamiento y la gestión de datos.

- Programas o Software as a Service (SaaS). Los usuarios de un programa acceden al mismo a través de una red de comunicaciones de forma que el programa se ofrece desde un centro de datos remoto. Ya no es necesario tener el programa instalado en el equipo localmente donde se quiere utilizar. Algunos ejemplos de este tipo de servicio son Google Mail, Microsoft Office Live, SalesForce.com, etc.

- Plataformas o Platform as a Service (PaaS). Las plataformas en la nube suelen ofrecer soporte para el desarrollo de programas a lo largo de todo el ciclo de vida del producto, lo que permite a los desarrolladores crear programas, utilizarlos y publicarlos directamente en la nube. No sOlo existen los programas finales sino que hay todo un entorno de desarrollo y programación para poder desarrollarlos, a diferencia del SaaS que solo ofrece el resultado final (el programa ya utilizable). Un claro ejemplo de PaaS es Google AppEngine.

- Infraestructuras o Infraestructure as a Service (IaaS). Los usuarios utilizan directamente los recursos físicos (infraestructura hardware) de los sistemas informáticos en la nube, tales como capacidad de proceso, almacenamiento, redes de comunicaciones y otros tipos de recursos. Uno de los usos habituales es el de la virtualización de ordenadores, de forma que se puedan crear pequeñas máquinas virtuales 
aisladas que permitan ser utilizadas y combinarse para adaptarse a los recursos que una o varias máquinas físicas puedan ofrecer. Algunos ejemplos de IaaS son los servicios EC2 de Amazon o Azure de Microsoft.

- Almacenamiento de datos o Data storage as a Service (DaaS). El servicio de almacenamiento virtual bajo demanda podría entrar dentro de los servicios IaaS aunque hoy en día cuenta con la suficiente importancia como para que algunos autores lo consideren como un tipo de servicio propio. No solo se refiere al almacenamiento (creación, acceso, modificación y borrado de archivos o de objetos), sino que también abarca la gestión de los datos almacenados a modo de base de datos. Algunos DaaS ofrecen servicios de almacenamiento de los datos a modo de tablas especiales que permiten escalar de forma ágil y facilitan sistemas de consulta y proceso de los datos guardados de manera eficiente, generalmente mucho mejor de lo que se podría hacer con sistemas RBDMs habituales. Algunos ejemplos de estos DaaS son Amazon S3, Google BigTable o Apache HBase.

Finalmente, es preciso conocer los diferentes modelos de despliegue que existen para los diferentes servicios de nube posibles. Para ello, se puede hacer referencia al informe (CCN 2013) del Centro Criptográfico Nacional Español sobre seguridad en el cloud de la guía 823 de seguridad en las TIC dentro del esquema nacional de seguridad (ENS). En este informe se identifican cuatro modelos de despliegue de servicios en la nube:

- Nubes públicas. Son aquellas que se ofrecen de forma abierta al público en general. Las infraestructuras y los recursos están en manos de terceros y los servicios pueden ser utilizados y compartidos por usuarios de múltiples organizaciones. Este tipo de nube es económica debido al pago por uso, pero se desconoce el tipo de servicios que se comparten en la nube con otras personas.

- Nubes privadas. Son de uso exclusivo de una sola organización y pueden ser gestionadas por la propia entidad, por un tercero o alguna combinación de los dos. Una nube privada puede ser interna, si está ubicada en las instalaciones del cliente, o externa, si los servidores se encuentran alojados en las instalaciones de un tercero.

- Nubes comunitarias. Se refiere a aquellas nubes que están preparadas para ser compartidas entre varias organizaciones que tienen algún elemento común (afinidad, seguridad, privacidad, cumplimientos normativos, etc.). Puede ser gestionada por las propias organizaciones participantes o por un tercero, y también pueden ser internas, si están implementadas en las instalaciones de uno de los participantes de la comunidad, o externas, si los servidores se encuentran alojados en un tercero.

- Nubes híbridas. Son aquellas en las que la infraestructura es una composición de dos o más tipos de nube (privada, comunitaria o pública) que mantienen su propia identidad pero unidas por una tecnología estandarizada que permita la portabilidad de las aplicaciones y de los datos.

\section{Considerando los riesgos y amenazas}

Aunque todos los servicios que se ofrecen desde la nube están expuestos a diferentes riesgos y vulnerabilides, quizá el tipo más importante sea el de SaaS, principalmente por su mayor nivel de implantación entre los diferentes tipos de usuarios. Desde preocupaciones por la seguridad de los datos almacenados, hasta la sobrecarga de trabajo que supone gestionar la seguridad de las infraestructuras virtuales o el miedo a ataques de hackers a través de las posibilidades que ofrecen los PaaS, los usuarios deben tomar conciencia de las posibles amenazas que supone utilizar este tipo de tecnologías para poder hacer un uso adecuado y fiable.

En un amplio estudio sobre las vulnerabilidades y amenazas de usar servicios en la nube, Subashini (2011) identifica algunos de los problemas principales y de los miedos por parte de los usuarios. Entre los principales se encuentra la seguridad de los datos, ya que, a pesar de las medidas de seguridad que ofrecen los proveedores de servicios en la nube, se debe proteger el acceso a los datos por parte de empleados de esos proveedores de forma que no puedan acceder a datos almacenados de los clientes. Esto implica el uso de técnicas de control 
fino a la hora de definir accesos y la aplicación de cifrados criptográficos de forma que la información guardada esté protegida y no sea de acceso directo para los empleados encargados de gestionar los servicios que la almacenan. De idéntica forma, el acceso entre los diferentes recursos de almacenamiento de datos y los sistemas habilitados para su proceso debe ser seguro, empleando conexiones SSL o TSL para que no puedan ser interceptados por ataques intermedios (Man-in-the-middle).

También Gartner (Brodkin, 2008) afirma en un estudio sobre riesgos en la nube que se deben tener en cuenta, entre otros aspectos, la consistencia y durabilidad de la información. Los datos no deben quedar inconsistentes o corruptos en la gestión de los mismos, por errores de cifrado, accesos concurrentes, transacciones duplicadas, etc.

Así mismo, el proveedor de servicios debe ofrecer un sistema de copia de respaldo que posibilite la recuperación de la información frente a desastres imprevistos o en caso de borrados accidentales. Tampoco hay que olvidar la observación detallada de la segregación de los datos, ya que, al tratarse de un entorno donde múltiples usuarios almacenan su información en recursos comunes, pueden existir fallos de seguridad que posibiliten el acceso a los datos de terceros sin su autorización.

Por último, es necesario observar con mucho detalle los aspectos legales del uso de los servicios en la nube ya que, en muchos casos, y en parte debido al tipo de datos almacenados y tratados, deben existir una serie de requisitos legales que posibiliten el uso de esos servicios. Alguno de los casos más habitual es la exigencia por parte de algunos países, como los de la Unión Europea (UE), de que ciertos datos clasificados como de nivel de protección media/ alta estén albergados físicamente en centros de datos que estén en la propia UE.

Es necesario para ello conseguir un acuerdo con el proveedor de servicios (Service Level Agreement o SLA) que garantice el lugar donde residirán siempre esos datos, independientemente de si están cifrados o segregados. También será necesario que los proveedores de servicios faciliten las investigaciones penales derivadas de actos inapropiados o ilegales en los que hayan intervenido servicios de la nube.

\section{Los mayores en la nube}

A pesar de que las personas mayores pueden utilizar todos los servicios de la nube con el mismo grado de eficiencia y aprovechamiento que cualquier otro usuario de edades inferiores, la mayor relevancia en investigación sobre el uso de la nube relacionado con las personas mayores se inscribe dentro del mundo de la asistencia médica y el desarrollo de servicios específicos que entrelacen las nuevas tecnologías de la nube con otras locales a las personas de forma que se pueda establecer un marco de atención personalizada.

Existen múltiples estudios que relacionan el uso de avances tecnológicos y la gerontología (Bronswijk y otros 2002), lo que en ocasiones se denomina gerontecnología, y que se fundamenta en cuatro pilares como la prevención de restricciones sobre las personas y soluciones, la compensación y asistencia al individuo, la atención y soporte y finalmente, la mejora y satisfacción de la calidad de vida. Para trabajar en estos cuatro conceptos, se utilizan disciplinas como los sistemas de comunicaciones, robótica, tecnologías de asistencia doméstica y monitorización a través de sensores y recolección de datos. Peterson y otros (2012) proponen el uso de estas tecnologías para la asistencia de personas que sufran una condición de demencia de modo que los sistemas de monitorización puedan detectar signos de esta enfermedad o se faciliten las comunicaciones entre enfermos y sus familiares, mejoren la gestión medica de los tratamientos y los seguimientos por parte de profesionales o ayuden en la asistencia doméstica de los propios enfermos.

Con la aparición de un número creciente de dispositivos que ofrecen nuevas posibilidades tecnológicas (instrumentos o monitores portables en la ropa o integrados en el cuerpo, etiquetas RFID, teléfonos y dispositivos inteligentes, etc.) se consigue integrar disciplinas biomédicas, mecánicas, eléctricas, de gestión de información y de nuevas tecnologías en comunicaciones. La nube hace posible que la cantidad de recursos necesarios para poder llevar a cabo todas estas nuevas tareas no recaiga directamente en los elementos físicos que residen en el hogar o en los dispositivos que el propio paciente pueda llevar consigo. 
Precisamente, estas tecnologías que intentan entrar en el contexto de la vida de las personas mayores para asistir y mejorar su vida están empezando a emplear los servicios en la nube para mejorar el rendimiento y construir aplicaciones, servicios y flujos de comunicación de forma rápida y eficiente. Estos sistemas de monitorización intensa (pervasive healthcare monitoring о $\mathrm{PH}$ ) sirven para velar por las personas mayores o pacientes crónicos que requieran una vigilancia constante $y$, a largo término, que tomen en consideración permanentemente sus constantes fisiológicas creando una alerta médica cuando sea requerido o almacenando esta información para su posterior análisis y seguimiento médico. Este tipo de servicios de almacenamiento y gestión de datos o de interacción entre paciente y centro médico ven las posibilidades que ofrece la nube como un gran aliado sobre el que basar la tecnología necesaria para realizar su función.

Zhou y otros (2012) definen un marco de trabajo para el contexto de uso de tecnologías de asistencia que abarquen las necesidades de una persona mayor (pervasive service computing), de forma que le ayuden en todas sus tareas diarias. Desde la monitorización del estado físico a través de sensores inalámbricos hasta el seguimiento en tiempo real de las constantes vitales o facilitar la comunicación con familiares y médicos puede ser creado, gestionado y dirigido a través de servicios en la nube.

También existen propuestas como la de Chen y otros (2011) donde se configura todo un ecosistema de servicios en la nube (IaaS, PaaS y SaaS) accesibles desde diferentes tipos de canales de comunicación (GSM 2G/3G/4G, wLAN/wimax, ZigBee, Bluetooth, radiofrecuencia, etc.) y que consigue crear un flujo de interacción entre el paciente, su hogar y los centros médicos. El diseño presentado permite la escalabilidad y ampliación de nuevos servicios bajo demanda de forma rápida.

En España, existen diferentes proyectos que tratan de aunar las tecnologías de la nube y el cuidado a las personas mayores. Uno de ellos es el proyecto Virtual Cloud Carer (vCC) de Gachet y otros (2012). Este proyecto tiene como principal misión crear nuevos servicios para mayores dependientes y enfermos crónicos usando tecnologías asocia- das a Internet y la computación en la nube. Esta misión se divide en diferentes objetivos, como los orientados a la tecnología, que persiguen desarrollar un sistema de telemonitorización y una plataforma de telecontrol para las personas dependientes y los cuidadores, tanto dentro de casa como en el exterior, empleando diferentes técnicas de comunicación.

Los objetivos sociales tratan de atajar la brecha que impide a los mayores y a las personas con enfermedades crónicas disponer de una calidad de vida adecuada, permitiendoles realizar sus tareas básicas diarias conociendo su estado físico en todo momento y también integrarlos y facilitar el acceso a las nuevas tecnologías de la información y la comunicación a través de Internet. Por último, también existen una serie de objetivos relacionados con la salud que consisten en proveer de un conjunto de metas que ayuden a los mayores a mantenerse físicamente activos y ayuden al personal médico a realizar un mejor seguimiento del cumplimiento de estas tareas que realizan las personas mayores en sus hogares.

\section{Caso práctico: Universidad para Mayores de la UJI}

En el caso concreto de la Universitat Jaume I de Castellón (UJI), el uso de servicios en la nube es bastante intenso en todos sus tipos. Cabe señalar que en el 2011 la Universitat Jaume I y Google firmaron un acuerdo de colaboración (UJI, 2013) fruto del cual se transfirieron algunos servicios corporativos a la nube de Google, por lo que todos los alumnos, profesores y trabajadores de la universidad disfrutan de las ventajas de este acuerdo.

No todos los miembros de la universidad hacen un uso idéntico de los servicios, sino que, en función del perfil de usuario, se pueden identificar unos usos más intensivos de los servicios. En concreto, se ha constatado que la mayoría de alumnos hacen un uso más habitual de los servicios SaaS y de IaaS, de forma idéntica al personal de carácter administrativo y docentes. Entre los investigadores predomina el uso de servicios IaaS. Cabe señalar que el uso de servicios PaaS no aparece prácticamente en los registros de acceso de los usuarios y su uso es 
residual, en muchos casos como parte de alguna prueba de concepto. En cualquier caso, estos perfiles de uso de los servicios por parte de los diferentes tipos de usuarios son bastante parecidos con algunos ejemplos publicados por parte de otras universidades que han adoptado intensamente el uso de servicios de la nube (Sultan, 2010).

Entre los servicios SaaS más utilizados destaca el uso de las Google Apps, una colección de programas accesibles vía web que incluyen aplicaciones como el correo electrónico (Gmail), mensajería instantánea (Gtalk y Hangout), calendario (Google Calendar) o un conjunto de suite ofimática (Google Docs, con procesador de textos, hoja de cálculo, presentaciones, etc.). Por otro lado, en los servicios IaaS se observa que el mayor uso es el del almacenamiento remoto, bien a través del propio servicio de Google (Drive) o de otros proveedores externos como DropBox o SkyDrive de Microsoft.

El uso intensivo de todos estos servicios por parte de los usuarios está acompañado por una exhaustiva campaña de formación por parte de la Universitat Jaume I a todos los niveles de perfil de usuario. Desde el departamento de recursos humanos se han realizado reiterados cursos de formación a los docentes y al personal de administración y servicios, mientras que los alumnos han visto cómo este tipo de herramientas y servicios han ido apa- reciendo recientemente en los temarios de las asignaturas que cursan.

La Universidad para Mayores de la Uji ha apostado desde los inicios de la aparición de esta tecnología por ofrecerla a los estudiantes como una herramienta más que forme parte del aprendizaje continuo a lo largo de la vida. Se ha considerado la nube como un concepto útil, que supone un buen servicio a los estudiantes y que familiariza con diferentes objetivos y conocimientos del ámbito tecnológico y educativo designados como primordiales en los programas nacionales y europeos de educación continua a lo largo de toda la vida (livelong learning).

Concretamente, se ha introducido el concepto y el uso de la nube en el currículo de todos los cursos de informática y nuevas tecnologías de los estudiantes de la Universidad para Mayores, se han realizado diversos talleres temáticos para estudiantes que ya han cursado sus asignaturas relacionadas con nuevas tecnologías y se han realizado acciones formativas y divulgativas como congresos, charlas y participación en proyectos de ámbito regional, nacional y europeo. También los miembros docentes y trabajadores han introducido el uso de los servicios de la nube en su labor diaria, principalmente con los servicios de SaaS de Google, aunque también con diferentes servicios IaaS para la gestión y el almacenamiento de datos. 


\section{Referencias}

Brodkin, J. (2008). Gartner: Seven cloud-computing security risks.

Bronswijk, J. V., Bouma, H., y Fozard, J. L. (2002). Technology for quality of life: an enriched taxonomy. Gerontechnology, 2(2), 169-172.

CCN - Centro Criptográfico Nacional. (2013). Seguridad en entornos cloud. Guía/norma de seguridad de las TIC (CCNSTIC-823). https://www.ccn-cert.cni.es/publico/seriesccn-stic/series/800-Esquema_Nacional_de_Seguridad/823-Seguridad-en-entornos-cloud/823-Cloud_Computing_ENS.pdf

Chen, K. R., Lin, Y. L., y Huang, M. S. (2011, octubre). A Mobile Biomedical Device by Novel Antenna Technology for Cloud Computing Resource toward Pervasive Healthcare. In Bioinformatics and Bioengineering (BIBE), 2011 IEEE 11th International Conference on (pp. 133-136). IEEE.

Dillon, T., Wu, C., y Chang, E. (2010, April). Cloud computing: Issues and challenges. In Advanced Information Networking and Applications (AINA), 2010 24th IEEE International Conference on (pp. 27-33). IEEE.

Gachet, D.., Aparicio, F., Ascanio, J. R., \& Beaterio, A. (2012). Innovative health services using cloud computing and internet of things. In Ubiquitous Computing and Ambient Intelligence (pp. 415-421). Springer Berlin Heidelberg.

Mell, P., y Grance, T. (2009). The NIsT definition of cloud computing. National Institute of Standards and Technology. Information Technology Laboratory, Version, 15(10.07), 2009.

Peterson, C. B., Prasad, N. R., y Prasad, R. (2012). The future of assistive technologies for dementia. Gerontechnology, 11(2), 195.

Subashini, S., \& Kavitha, V. (2011). A survey on security issues in service delivery models of cloud computing. Journal of Network and Computer Applications, 34(1), 1-11.

Sultan, N. (2010). Cloud computing for education: A new dawn? International Journal of Information Management, 30(2), 109-116.

UJI (2013) http://google.uji.es/es/la-uji-migra-la-nube (Enlace visitado y revisado el 13 de julio del 2013)

Vaquero, L. M., Rodero-Merino, L., Caceres, J., y Lindner, M. (2008). A break in the clouds: towards a cloud definition. АСм SIgCOмм Computer Communication Review, 39(1), 50-55.

Zhou, J., Su, X., Ylianttila, M., y Riekki, J. (2012, enero). Exploring pervasive service computing opportunities for pursuing successful ageing. In Grid and Pervasive Computing Workshops (pp. 73-82). Springer Berlin Heidelberg. 
